\title{
Coronary Illness Prediction Using Random Forest Classifier
}

\author{
Rekha $\mathrm{G}^{\mathrm{a}, 1}$, Shanthini $\mathrm{B}^{\mathrm{a}}$ and Ranjith Kumar $\mathrm{V}^{\mathrm{b}}$ \\ a Department of CSE, St. Peter's Institute of Higher Education \& Research, \\ Chennai, TN, India \\ ${ }^{\mathrm{b}}$ Department of Mechanical Engineering, Sri Sairam Engineering College, \\ Chennai, TN, India
}

\begin{abstract}
Heart diseases or Cardiovascular Diseases (CVDs) are the main cause of death on the planet throughout the most recent years and become the most dangerous disease in India and the entire world. The UCI repository is utilized to calculate the exactness of the AI calculations for foreseeing coronary illness, as knearest neighbor, decision tree, linear regression, and support vector machine. Different indications like chest pain, fasting of heartbeat, etc., are referenced. Large datasets, which are not available in medical and clinical research, are required in order to apply deep learning techniques. Surrogate data is generated from Cleveland dataset. The predicted results show that there is an improvement in classification accuracy. Heart disease is one of the most challenging diseases to diagnose as it is the most recognized killer in the present day. Utilizing AI algorithms, this paper gives anticipating coronary illness. Here, we will use the various machine learning algorithms such as Support Vector Machine, Random Forest, KNN, Naive Bayes, Decision Tree and LR.
\end{abstract}

Keywords. Coronary artery disease, Decision tree, $\mathrm{K}$ nearest neighbor; SVC, Logistic Regression, Naïve Bayes, Accuracy

\section{Introduction}

Cardiovascular disease is the most recognized killer in the present world. Consistently an excessive number of individuals are kicking the bucket because of heart illness. CAD can emerge because of lacking blood supply to courses. The two most common cardiac emergencies are a heart attack and myocardial infarction.

Heart disease describes a group of conditions that affect heart. Heart diseases include:

- Arrhythmias

- Congenital Heart Defects

- Heart valve disease

The aim of this study is to achieve accuracy so that it can predict a heart attack. Ages, sex, blood pressure, cholesterol, chest pain, heart rate, and so on are risk factors.

\footnotetext{
${ }^{1}$ G.Rekha, Department of CSE, St. Peter's Institute of Higher Education \& Research, Chennai, TN, India; Email: rekhabensy@gmail.com.
} 


\section{Literature Review}

Proposed a GUI created in python by utilizing tkinter to produce a basic dialog box which takes contribution for all the qualities essential for assessment [1]. Advanced AI techniques, for example, neural organizations and support vector machines are connected to NB, linear regression [2]. Portrays the hereditary calculation (GA)-SVR with genuine esteem GAs machine learning strategy [3]. Presents a model for predicting the next day's stock price. The interaction of intelligent agents was used in an AI model for this task [4].

A forecasting model for tourists is the proposed make use of LLSSVR (logarithm leastsquares support vector regression) technologies [5]. Analyzes performance of ELMs to those of classification, support vector machine, and regression applications [6].

\section{Methodology}

In this paper, we have utilized our dataset for applying different machine learning algorithms for recognizing if an individual has coronary illness or not.

\subsection{Data Collection}

The data is acquired from the Cleveland Heart Disease database at UCI Repository. There are 14 attributes in the dataset. The dataset is narrated as follows:

i. Age: describes the age.

ii. Sex: describes the gender identity; 1 - male, 0 - female.

iii. (cp): describes the form of chest.

iv. (trestbps): describes the bp.

v. (chol): describes the serum cholesterol.

vi. FBS: describes the fasting blood sugar (1 for true \& 0 for false).

vii. (restecg): describes the ECG results

viii. (thalach): describes the heartbeat rate.

ix. (exang): describes the angina.

x. Oldpeak: describes the depression mood.

xi. (slope): describes the slope.

xii. (ca): describes the blood vessels.

xiii. (thal): describes thal feature (3 for normal, 6 for fixed defect, 7 for reversible effect).

xiv. (target): describes target class.

\subsection{Algorithms Applied}

Gaussian Naive Bayes, Decision tree, SVM and Random Forest, KNN.

\section{Results}

\subsection{Correlation Matrix}

From the Figure 1, we can see that a few highlights are exceptionally related and some are definitely not. 


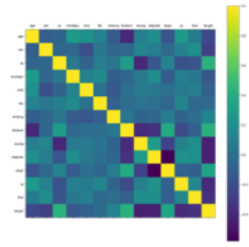

Figure 1. Features of Correlation Matrix

\subsection{Histogram}

It is a statistical tool for the portrayal of the appropriation of the data set in Figure 2.

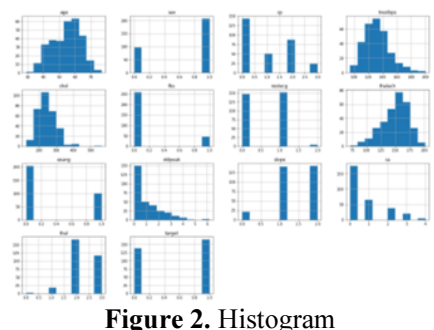

Figure 2. Histogram

Figure 3, Out of all the patients 165 patients actually have coronary illness. Now also visualize.

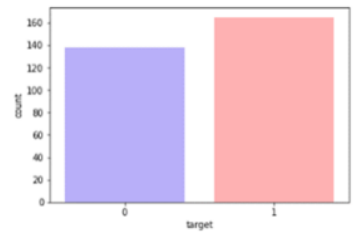

Figure 3. Count of the number of patients having heart disease (target $=1$ ) Figure 4, classifying target variable between male and female and visualize the result.

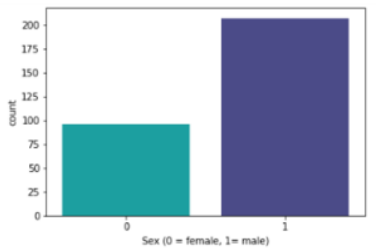

Figure 4. Count of both gender having heart disease

Figure 5, Scatter plot between "Maximum Heart Rate" and "Age" is shown below.

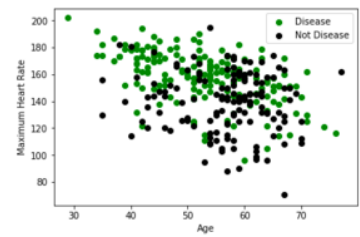

Figure 5. The Scatter plot between Age and Maximum heart rate 
Exploratory Data Analysis:

The process of analyzing data, planning a new plan, test hypothesis, checking assumptions, and uncovering a hidden pattern is known as exploratory data analysis.

The Bar plot for target class (different features):

It is vital that the dataset we are utilizing ought to be pre-prepared and cleaned. This Figure 6 shows the tally of each target class.

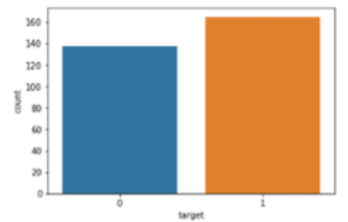

Figure 6. Count versus Target Feature

The chart above portrays the distribution of target versus count class, that is utilized to anticipate if an individual has coronary illness $(0=$ no coronary illness, $1=$ coronary illness).

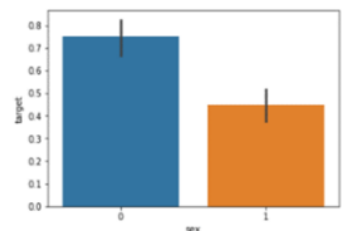

Figure 7. Target versus Sex Feature

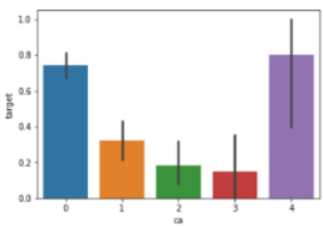

Figure 8. Target versus CA Feature

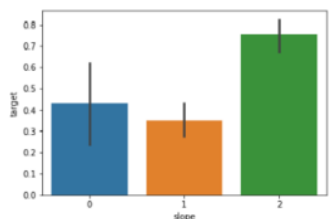

Figure 9. Target versus Slope Feature

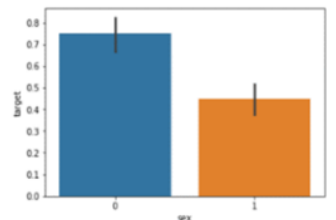

Figure 11. Target versus Restecg Feature

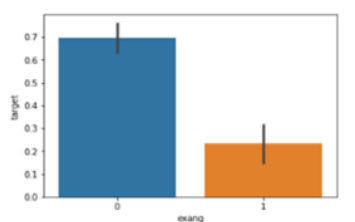

Figure 10. Target versus Exang Feature

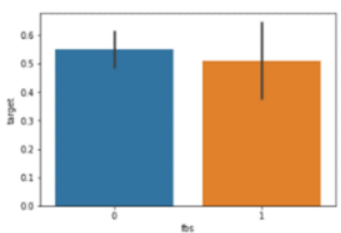

Figure 12. Target versus Fbs Feature 


\section{Machine Learning Algorithms}

\subsection{Logistic Regression}

The supervised learning algorithm logistic regression is utilized to anticipate the parallel type of objective variable. In this paper, we achieved the accuracy of $85.25 \%$ by using this model.

\subsection{Nä̈ve Bayes Classifier}

A Naive Bayesian classifier has equivalent execution with DT and other selected classifiers. In this paper, we achieved the accuracy of $85.25 \%$ by using this classifier.

\subsection{K Nearest Neighbors}

This calculation is utilized when the measure of information is enormous and there are non-decision boundaries between classes. In this paper, we achieved the accuracy of $67.21 \%$ by using this classifier.

\subsection{Support Vector Classifier}

This classifier is based on dataset containing features and labels.

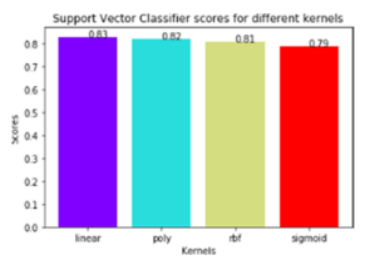

Figure 13. Support Vector Classifier scores.

This graph shows that the linear kernel is having the highest accuracy of $83 \%$ by using this model.

\subsection{Decision Tree Classifier}

It can be used to solve problems including regression and classification.

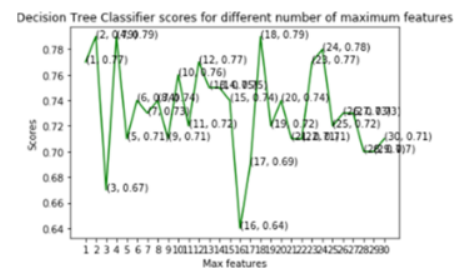

Figure 14. Decision Tree Classifier scores

This graph shows the line graph from which we observed that the maximum accuracy is $81.97 \%$.

\subsection{Random Forest}

It creates decision trees from data samples chosen at random, makes expectations for each tree, and selects the best solution. 
Table 1. Accuracy table

\begin{tabular}{|l|l|}
\hline Algorithms & Accuracy \\
\hline Gaussian NB Classifier & $85.25 \%$ \\
\hline K Nearest Neighbors Classifier & $67.21 \%$ \\
\hline Logistic Regression & $85.25 \%$ \\
\hline Decision Tree Classifier & $81.97 \%$ \\
\hline Support Vector Classifier & $83 \%$ \\
\hline Random Forest Classifier & $95.08 \%$ \\
\hline
\end{tabular}

This table 1 shows the Random Forest Classifier which we observed that the maximum accuracy is $95.08 \%$

\section{Conclusion}

This paper includes determining coronary illness dataset with legitimate data processing and execution. With an accuracy of 95 percent, Random Forest Classifier is perhaps the most successful of all the machine learning algorithms.

\section{Future Enhancements}

This may permit new calculation improvement to be performed off-site utilizing distributed computing programming, and afterward got back to the clinical setting as applications program interfaces (APIs) for PCs, cell phones and tablets.

\section{References}

[1] Sharma, V., Yadav, S., \& Gupta, M. (2020). Heart Disease Prediction using Machine Learning Techniques. 2020 2nd International Conference on Advances in Computing, Communication Control and Networking (ICACCCN), 177-181. https://doi.org/10.1109/ICACCCN51052.2020.9362842

[2] Hong, W.-C., Dong, Y., Chen, L.-Y., \& Wei, S.-Y. (2011). SVR with hybrid chaotic genetic algorithms for tourism demand forecasting. Applied Soft Computing, 11(2), 1881-1890. https://doi.org/10.1016/j.asoc.2010.06.003

[3] Zarandi, M. H. F., Hadavandi, E., \& Turksen, I. B. (2012). A hybrid fuzzy intelligent agent-based system for stock price prediction. International Journal of Intelligent Systems, 27(11), 947-969. https://doi.org/10.1002/int.21554

[4] Pai, P.-F., Hung, K.-C., \& Lin, K.-P. (2014). Tourism demand forecasting using novel hybrid system. Expert Systems with Applications, 41(8), 3691-3702. https://doi.org/10.1016/j.eswa.2013.12.007

[5] Sun, X., Sun, W., Wang, J., Zhang, Y., \& Gao, Y. (2016). Using a Grey-Markov model optimized by Cuckoo search algorithm to forecast the annual foreign tourist arrivals to China. Tourism Management, 52, 369-379. https://doi.org/10.1016/j.tourman.2015.07.005

[6] Al-Musaylh, M. S., Deo, R. C., \& Li, Y. (2020). Electrical Energy Demand Forecasting Model Development and Evaluation with Maximum Overlap Discrete Wavelet Transform-Online Sequential Extreme Learning Machines Algorithms. Energies, 13(9), 2307. https://doi.org/10.3390/en13092307Sangya Ware, Shanu K Rakesh, Bharat Choudhary, Heart Attack Prediction by using Machine Learning Techniques, IJRTE, ISSN: 2277-3878, Vol. 8 Issue 5, January 2020 . 